\title{
Radiation Oncology Policy for Risk reduction from novel COVID-19 Pandemic in Radiation Oncology Department
}

\section{Dr Bushra Rana}

March 27,2020

Correspondence should be directed to

Dr Bushra Rana

Team Net Medical

1135 Heather stone drive,

Fredericksburg, VA 22407

202-536-9399 (T)

Bushra.rana@teamnetmedical.com

\begin{abstract}
This brief policy is written after experience treating COVID-19 positive radiation therapy patients to reduce risk to therapy staff and patients in radiation oncology department. It is important to prioritize the safety of staff and non-infected patients while ensuring the continuation of radiation oncology services. Radiation therapists have sustained contact with covid-19 patients in an enclosed vault. Protocols for correct disinfecting of equipment and room and therapists following methods for less transmission of virus is crucial. This policy covers prevention methods from COVID-19 transmission from patient to patient, patient to staff, staff to patient and staff to staff as follows
\end{abstract}
A. Risk reduction by screening and preparing staff and rooms
B. Radiation Therapist Policy for COVID-19 positive patient with CCC (Critical Cancer Care)

Keywords: Radiation oncology, COVID-19, Radiation therapist COVID-19 policy, Radiation oncology departmental Policy 


\section{A. Risk reduction from COVID-19 in radiation oncology}

\section{IMPORTANT:}

\section{COVID-19 Positive patient or suspected positive should be considered the same}

\section{Patient time in the department should absolutely be minimized.}

\section{DONOT treat COVID-19 positive patients whose treatment can be delayed for a month till they recover from COVID-19.}

Infection Control Visit: It is suggested to have an infection Control personnel visit to the radiation oncology department and walk through the patient flow to suggest any changes to the current disinfecting process.

\section{Ports of Entry into the department}

(1) Restrict ports of entry to one each for patient and staff. Its recommended to keep the staff entry separate from the patient entry.

(2) Identify an exam room that would be labeled and used for possible COVID-19 or COVID-19 positive patients.

\section{Prep Waiting Areas}

Move waiting area chairs 6 feet apart.

Waiting areas should not have carpeting or non-impervious upholstered chairs.

Disinfect waiting area once every day and after a COVID-19 positive patient visit.

\section{Staff Screening Daily}

Screen staff at department entrance for fever, cough, upper and lower respiratory symptoms. Upper respiratory symptoms include sore throat, runny nose etc.

If symptoms are present, send the staff home and notify infection control for COVID-19-19 testing OR instruct staff to get COVID-19 testing. Follow-up test results and log dates of sending staff home.

For staff showing symptoms:

If staff with symptoms opted not to get tested, they should be symptom free for $72 \mathrm{hrs}$. before coming to work from quarantine.

Staff who tested negative for COVID-19, need to be symptom free for $48 \mathrm{hrs}$. before returning to work. 
All staff including front desk engaging in direct patient care of a COVID-19 positive/COVID-19 suspected should wear PPE (gown, glove, face shield, N95 mask with eye protection).

All staff shall get in-service (or watch an approved video) on how to don/doff PPE. Log in-service attendance.

\section{Patient screening daily}

All patients entering the cancer center shall wear surgical mask at all times.

Screen patient at the door entrance to the department for fever, cough, upper and lower respiratory symptoms. Upper respiratory symptoms include sore throat, runny nose etc. Ask patients about their recent travel and if they had any exposure to

Patient visitors not allowed in the department. If patient needs assistance from family limit one visitor to the waiting area. Ensure the visitor gets screened.

Patient and visitor have to wash their hands after entering the department. Ensure hand washing instructions are posted and verbally told to the patients. OR Patient gel in before going in and before going out. Gel instructions are the same as washing the hands and should be posted.

\section{If patient has COVID-19 symptoms (possible COVID-19),}

-Give patient a mask to wear.

-Send the patient for a COVID-19 test. If patient comes positive delay treatment if possible, till patient recovers.

-All staff that comes in contact with the patient (including front desk) should take droplet precautions. Droplet precautions for staff is surgical mask, gloves, eye protection and gown.

-Wipe down any area patient has touched when entering the facility. Follow wiping down rules (wearing gloves and doffing). 


\section{COVID-19 Positive Patients that have to wait in the cancer center}

If COVID-19 patient has to wait in the cancer center for any reason, patient shall be isolated in the COVID-19 dedicated isolation exam room with door closed.

Use negative pressure room/area if available. For methods for temporary negative pressure isolation, go to https://www.teamnetmed.com/resources.

After patient leaves the isolation room, the door to the room should be closed for an hour and then terminal disinfected*

Any staff entering the room shall wear the appropriate PPE when entering the room.

Patient should not be waiting in waiting rooms or any rooms with carpeting or non-impervious upholstered chairs.

\section{Critical Cancer Cases (CCC) and Non-Critical Cancer Cases (NCCC)}

Critical Cancer Cases (CCC): To prioritize the safety of staff and other patients in radiation oncology, it is suggested to treat only those known coronavirus cases who are Critical Cancer Cases. According to preprint 3-17-2020 "Radiation Oncology and COVID-19, Rao et al." "Critical Cancer Cases (CCC) are those cases where a delay of treatment for 14 days or greater may result in a loss of life or a permanent loss of organ. Examples of CCC include but are not limited to cord compression, superior vena cava syndrome, life threating tumor bleeding, airway obstruction by tumor, and highly symptomatic brain metastases. Other circumstances may also be CCC at the discretion of the treating physician"

Non-Critical Cancer Cases (NCCC): If the patient has NCCC and diagnosis of coronavirus or is under quarantine for coronavirus, then the patient shall not be seen for consult or radiation therapy until the waiting period._According to preprint 3-17-2020 "Radiation Oncology and COVID-19, Rao et al." "NonCritical Cancer Cases (CCC) are those cases where a delay of treatment for 14 days or greater is unlikely to result in a loss of life or a permanent loss of organ. Examples of CCC include but are not limited to: breast cancer, prostate cancer, rectal cancer, lung cancer, anal, head and neck, brain tumors, sarcomas and any non-symptomatic metastatic disease.

Coronavirus waiting period is defined as a treatment delay for 14 days or until the patient quarantine is completed, or the patient is non-infectious as defined by the CDC guidance.

\section{General radiation planning guidelines}

For COVID-19 positive CCC minimize fractions as much as possible. Use single fraction treatment for palliation.

Hypo fractionate as much as possible. 


\section{TELEHEALTH}

If the department is approved for tele health all OTVs and follow-ups should be done through telemedicine.

New consults should be evaluated case by case for tele medicine. In some instances, new patient consult has to be done in person. Patient's time should be kept at the minimum during on site consult.

\section{TELEWORK}

Unless you are required to be onsite to perform your work, work from home. Telework shall be done in an effort to preserve staff to cover during COVID-1919 outbreak or a COVID-1919 exposure in the department.

All remote accesses to record and verify system, planning system and network drive need to be made available for telework.

Have staff test their laptops/computers at home before starting telework.

Dosimetrist: For sites with more than one dosimetrist, one shall remain on site rest of the dosimetrists shall perform telework from home for two weeks. Switch dosimetrist after 14 days. Tele dosimetrists shall be available by phone during working hours. Dosimetrists shall be a backup for the therapists to treat on the machine if short staffed or therapist staff has to quarantine.

Physicist: For sites with more than one physicist, one physicist shall stay onsite and rest perform telework for two weeks. Switch after 14 days. Physicist shall be available by phone during working hours. Physicist shall have the added responsibility to do treatment planning, in case dosimetrist have to cover for quarantined therapists.

Physician: For site with more than one physician, one physician shall perform telework to preserve staff in case a physician has to quarantine.

Admin Staff: One admin staff shall do telework in an effort to preserve staff during COVID-19 outbreak or a COVID-19 exposure in the department 


\section{B. Radiation Therapist Policy for COVID-19 positive patient with CCC (Critical Cancer Care)}

\section{$\underline{\text { Treatment Vault }}$}

(1) Dedicate a vault for COVID-19 positive patient if more than one Linacs in the department.

(2) Negative pressure

-Use a negative pressure vault if available.

-Check with your maintenance department to see if Negative pressures can be created in the vault. Go to https://www.teamnetmed.com/resources for 'Methods for temporary negative pressure isolation. For ventilation configuration for negative pressure check Table 1.

(3) All extra devices/equipment in the vault shall be put away in closed cabinets or taken out of the room before treatment.

\section{Personal protective equipment (PPE) for therapy staff}

(1) Full PPE should be used by the therapy staff treating COVID-19 positive/COVID-19 suspected patients

(2) Given the proximity of patients to therapy staff and the sustained contact with patient, modified Airborne/Contact precautions should be used. Therapy staff shall take 'airborne precautions' which includes N95 respirator, gloves, gown, eye protection /face shield at all times when attending the COVID-19 patient. All staff shall get an in-service (or watch an approved video) on how to don/doff PPE.

(3) Every therapist should go through an in-house fit testing by wearing the N95 and checking if they can smell while breathing through it.

(4) Use P100 respirators for centers that have them for therapists and have been tested annually. Make sure to order extra filters for each respirator. Packed filters have a life of 5 years. Open filters should be replaced after six (6) months. Even when respirators have not been used, they keep absorbing dust and particles, so filters should be replaced.

(5) Replace filter (for P100) and mask (N95) once it is becoming difficult to draw a breath, or if smelly vapors are making their way through the mask.

(6) Face shields should be dipped in bleach and water to clean. 


\section{$\underline{\text { Radiation Treatment }}$}

(1) COVID-19 positive or suspected COVID-19 positive patient that fall in the CCC (Critical Cancer Care) category shall be treated at the end of the day, after hours with minimal staff in the department.

(2) Therapy staff shall have a full PPE on before escorting the patient into the department. All radiation therapy staff associated with the treatment shall be wearing airborne PPE. N95, face shield, eye wear, gloves, gown.

(3) Patient shall call ahead and stay in their car till they are called by the radiation therapist for treatment.

(3) Patient needs to removes dentures or any similar items in the car or leave it at home. Give patient a surgical mask and gloves (hand sanitizer before wearing the gloves)

(4) COVID-19 patient shall enter from the dedicated entrance that is the closest to the treatment machines.

(5) Disinfect* every area, door knobs, chair rails that COVID-19 patient would touch entering the department.

(6) Verbal check in and time out at treatment machine still required.

(7) For patients with aquaplast immobilization - have the patient wear a surgical mask inside the aquaplast face mask if the patient can tolerate it. Otherwise place mask on the exterior of aquaplast mask.

(8) Close LINAC door for 1 hour after treatment. After 1 hour, radiation therapy staff shall disinfect devices, table, linac head and imaging arms and floor with disinfectant wipes. Do not use disinfectant spray on the linear accelerator or any place that has electronics.

(9) Call environmental services and alert them. Terminal clean the room and linac before treating next day

(10) Dedicate same two therapists to treat COVID patients every day.

\section{Setting up the patient for treatment}

(1) Avoid possibility of leaning above the patient. Find an alternative to an AP SSD if possible, e.g setup patient with table height/table parameters/table indexing and Lat SSD/tattoo. If you have OBI/CBCT, use imaging for accurate setup.

(2) Keep extra time on schedule for patients with breathing/cough symptoms. Reimaging is required as many times it take to get the right setup. If patient cough after setup, reimage to confirm accuracy. 
(3) Wash hands or use hand disinfectant and change into new gloves before using the treatment console.

\section{Leave vault empty after treatment and before disinfecting}

If you don't have a negative pressure vault (which most are vaults are not), do airborne precautions. Leave room empty for 45 minutes after treating COVID-19 positive/possible COVID-19 patient and then staff can go in to clean/disinfect the devices and the room. Terminal disinfecting of the room shall be done after

\section{Disinfecting the linear accelerator}

Disinfect the linac head and OBI/Portal imager arms with disinfectant wipes after treating the COVID-19 suspected/positive patient. DONOT use bleach or disinfectant spray on the linac.

\section{Protecting and disinfecting Immobilization devices and Linac}

(1) Patient specific devices like molds, vac-lok, facemasks, custom headrests etc

-After treating the patient change gloves (use right doffing of gloves procedure) before cleaning devices.

-For vaclocks, custom head rests and mold, put them in a bag and then use on the patient. When finished using discard the bag, wipe down the mold and rebag it before hanging on the rack. Use a new clear plastic bag every day.

-Do not use moldable headrests which have cloth/fibrous exteriors.

-It is preferred that the COVID-19 or possible COVID-19 patient wear a surgical mask under the aquaplast facemask. If the patient cannot tolerate, have them wear it above the aquaplast face mask. Disinfect the aquaplast facemask after treatment and place it in a clear bag for storage.

(2) Non patient specific devices

For treatments where patients are breathing into a device, evaluate if you can avoid this technology and treat without it for COVID-19 positive or possible COVID-19 patients. Do breathe hold technique instead of respiratory gating if possible.

Use plastic covers on the breast board, wrap the head rest and other immobilization devices. After use, throw the used bag and disinfect the device thoroughly and re-bag in a clean clear bag. Use a new bag every day. 


\section{Aerosolizing procedure for COVID-19 positive}

Procedures like anesthesia and intubation for paeds are high risk, where a cough could be induced. Natural airway anesthesia is preferred if possible.

Staff should adopt 'airborne precautions' which include N95 or P100 respirators, facial shield, respirators suits PAPR or CAPR and gloves.

\section{$\underline{\text { Ventilator patients }}$}

Patients on ventilators shall not be treated unless absolutely necessary for an aggressively growing tumor that cannot wait for $2 / 3$ weeks

If have to treat, intubate in a negative pressure room and bring them down to the vault. Have anesthesia team present monitoring aerosol event.

Table 1: Ventilation configuration for negative air pressure (MN department of health -Airborne Infectious disease management)

\begin{tabular}{cccc}
\hline ACH & $\begin{array}{c}\mathbf{9 0 \%} \\
\text { EFFIEIECY }\end{array}$ & $\begin{array}{c}\mathbf{9 9 \%} \\
\text { EFFIIIEMGY }\end{array}$ & $\begin{array}{c}\mathbf{9 9 . 9 \%} \\
\text { EFFIIIENG }\end{array}$ \\
\hline $\mathbf{2}$ & 69 & 138 & 207 \\
\hline $\mathbf{4}$ & 35 & 69 & 104 \\
\hline $\mathbf{6}$ & 23 & 46 & 69 \\
\hline $\mathbf{8}$ & 17 & 35 & 52 \\
\hline $\mathbf{1 0}$ & 14 & 28 & 41 \\
\hline $\mathbf{1 2}$ & 12 & 23 & 35 \\
\hline $\mathbf{1 5}$ & 9 & 18 & 28 \\
\hline $\mathbf{2 0}$ & 7 & 14 & 21 \\
\hline $\mathbf{5 0}$ & 3 & 6 & 8 \\
\hline
\end{tabular}

Modified from Table B.1, CDC Guidelines for Environmental Infection Control in Health-Care Facilities, $2003^{5}$

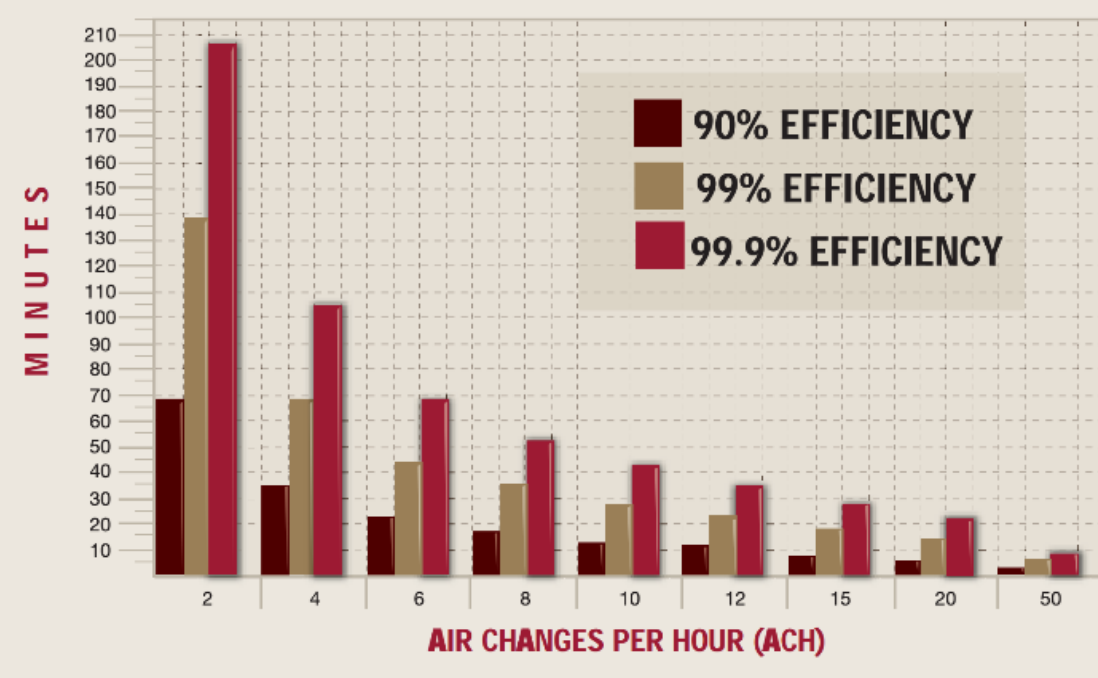

Perfect mixing of air is assumed. For rooms with stagnant air spaces, the time required may be much longer than shown. This is intended only as an approximation and is for ideal ventilation configurations. 


\section{Conclusion}

It's imperative to prioritize the safety of staff and noninfectious patients by taking precautions and following methods to reduce COVID-19 transmission. Above protocol has been implemented in a few radiation oncology centers around the country. This policy does not include details on PPE, droplet/airborne precautions, donning/doffing PPE which is very important to prevent transmission.

\section{References}

1. Rao et al. A radiation oncology departmental policy for the 2019 Novel Coronavirus (COVID-19) pandemic

2. MN department of health, office of emergency preparedness Airborne Infectious Disease management: Methods of temporary Negative Pressure isolation

3. www.cdc.gov

*Terminal disinfection of coronavirus exposed room: Terminal cleaning is the thorough cleaning/disinfection of all surfaces including floors and re-useable equipment within the facility following an outbreak or increased incidence of infection. Usually environmental services would perform the terminal cleaning. Ensure to empty the room out before taking the infected patient in the room. Therapist should still disinfect the immobilization devices and equipment including the linac before the terminal cleaning 